\title{
Rational synthesis of atomically precise graphene nanoribbons directly on metal oxide surfaces
}

\author{
M. Kolmer ${ }^{1}$, A.K. Steiner ${ }^{2}$, I. Izydorczyk ${ }^{3}$, W. Ko ${ }^{1,4}$, M. Engelund ${ }^{5}$, M. Szymonski ${ }^{3}$, A.-P. Li1, ${ }^{1, *}$, K. Amsharov ${ }^{2,6 *}$ \\ ${ }^{1}$ Center for Nanophase Materials Sciences, Oak Ridge National Laboratory, Oak Ridge, TN 37831, USA. 2Department of Organic Chemistry, Friedrich Alexander University \\ Erlangen-Nuremberg, Nikolaus-Fiebiger Str 10, 91058 Erlangen, Germany. ${ }^{3}$ Centre for Nanometer-Scale Science and Advanced Materials, NANOSAM, Faculty of Physics, \\ Astronomy and Applied Computer Science, Jagiellonian University, Łojasiewicza 11, 30-348 Kraków, Poland. ${ }^{4}$ Department of Physics and Astronomy, The university of \\ Tennessee, Knoxville, TN 37996, USA. ${ }^{5}$ Espeem S.A.R.L. (espeem.com), 9 rue de Haut-Fournaux, L-4365 Esch-sur-Alzette, Luxembourg. ${ }^{6}$ Institute of Chemistry, Organic \\ Chemistry, Martin-Luther-University Halle-Wittenberg, Kurt-Mothes-Strasse 2, 06120 Halle, Germany. \\ *Corresponding author. E-mail: apli@ornl.gov (A.-P.L.); konstantin.amsharov@fau.de (K.A.)
}

Atomically precise graphene nanoribbons (GNRs) attract great interest because of their highly tunable electronic, optical, and transport properties. However, on-surface synthesis of GNRs is typically based on metal-surface assisted chemical reactions, where metallic substrates strongly screen their designer electronic properties and limit further applications. Here, we present an on-surface synthesis approach to forming atomically precise GNRs directly on semiconducting metal oxide surfaces. The thermally triggered multistep transformations preprogrammed in our precursors' design rely on highly selective and sequential activations of $\mathrm{C}-\mathrm{Br}, \mathrm{C}-\mathrm{F}$ bonds and cyclodehydrogenation. The formation of planar armchair GNRs terminated by well-defined zigzag ends is confirmed by scanning tunneling microscopy and spectroscopy, which also reveal weak interaction between $\mathrm{GNRs}$ and the rutile $\mathrm{TiO}_{2}$ substrate.

Atomically precise GNRs $(1,2)$ can host nontrivial electronic structures (3-5), magnetic edge states (6-8), and coupled spin centers (9-11). These properties make GNRs promising candidates as functional building blocks for applications in emerging quantum information science (QIS) (12). The rational synthesis of GNRs have been achieved by designing organic molecules to undergo surface-catalyzed reactions under ultrahigh vacuum (UHV) conditions after deposition on a crystalline substrate $(13,14)$. The commonly used strategies are based on intramolecular carbon-carbon (C-C) bond formations leading to annulation through thermally triggered cyclodehydrogenation reactions (15), which are preceded by intermolecular C-C coupling (polycondensation of precursor units, see Fig. 1A).

A two-step approach reported by Cai et al. (16) works reliably on noble metal surfaces. However, the high density of low-energy electronic states inherent to a metal substrate does not allow for sufficient electronic decoupling of the synthesized GNRs, which necessitates a transfer onto semiconducting or insulating substrates for detailed characterization or prototypical device fabrication. So far, these transfer processes have been performed locally by scanning tunneling microscopy (STM) tip-induced manipulation of single molecules $(6,17)$ or globally either by in-situ intercalation of a decoupling layer (18) or ex-situ by using wet-chemistry processing (19-21). For spintronic and QIS applications, which rely on atomically defined nanostructures and their subtle interactions, establishing reliable on-surface chemistry methods for nonmetallic substrates is a desirable solution.
In this regard, the versatile chemical activity of transition metal oxides that finds use in heterogeneous catalysis is attractive. In particular, aryl halide precursors undergo surface-assisted polycondensation on rutile $\mathrm{TiO}_{2}$ surfaces through thermal activation of carbon-bromine (C-Br) or carbon-iodine (C-I) bonds (Fig. 1A) (22-24). However, for intramolecular annulation, a lack of catalytic activity of the metal oxide surface demands alternatives for the cyclodehydrogenation reaction (22). Thus, direct synthesis of planar GNRs by mimicking the two-step triggering strategy used for metal substrates could not be accomplished on nonmetallic substrates. Previously, we demonstrated that intramolecular aryl-aryl coupling in polycyclic aromatic hydrocarbon precursors can be achieved by the activation of carbon-fluorine (CF) bonds on various metal oxides (25-29), including the $\mathrm{TiO}_{2}$ surface (30). The high efficiency of this approach enabled onsurface synthesis of two confined nanographenes through thermally initiated sequential cyclodehydrofluorination reactions (Fig. 1B).

However, the formation of extended $\mathrm{sp}^{2}$ carbon systems, like GNRs, by combining the reported polycondensation and cyclisation processes, remains an open challenge. Here, we report a methodology for rational precursor design and direct synthesis of atomically precise GNRs on the rutile $\mathrm{TiO}_{2}(011)$ $(2 \times 1)$ surface. The multistep on-surface synthesis programmed in our precursors consisted of sequential polycondensation, cyclodehydrofluorination, and cyclodehydrogenation reactions. These thermally-triggered processes led to formation of finite-length 7-armchair 
graphene nanoribbons (7a-GNRs) terminated by well-defined zigzag ends (31). Such finite 7a-GNRs are open-shell character molecules with a single electron localized at each end (7, 32). Our scanning tunneling microscopy and spectroscopy (STM/S) analysis of this model system revealed weak screening of the intrinsically magnetic end-states by substrate electrons, which is a prerequisite for spintronic applications.

To realize the on-surface synthesis strategy, we used 10,10"-dibromo-1',4'-difluoro-9,9':10',9"-teranthracene

(DBDFTA, 1, see details of organic synthesis and characterization in Supplementary Materials) presented in Fig. 1C. The rational design of (1) was imbued with our conception of controlled consecutive reactions. As shown previously (22), terminal bromine-atom functionalization ensured the polycondensation step, which was facilitated by nonplanar geometry of the teranthracene chassis. Such precursor conformation weakened interactions with the substrate to allow sufficient precursor mobility and minimized steric hindrance during inter-molecular $\mathrm{C}-\mathrm{C}$ bond formation. As the intermolecular coupling reactions on $\mathrm{TiO}_{2}$ can be optimized (24) or achieved by other approaches (33), our main focus was on the effective transformation of resulting oligoanthracenes into planar GNRs.

Following our previous reports, we initiated this process by metal oxide surface-induced C-F bonds activation (30). During rational design of precursors, the substrate-activated $\mathrm{C}$-F bond must be paired with an electron-rich $\pi$-system in order to undergo effective cyclodehydrofluorination (34). For larger $\pi$-extended systems, the cyclization reaction proceeded more effectively (35). In the case of compact nanographenes, this effect could be used to implement self-accelerating, consecutive reactions with each subsequent cyclization step proceeding faster because of the increased size of the corresponding $\pi$-system involved (Fig. 1B). However, the presence of electron-withdrawing $\mathrm{F}$ atoms in a given aryl group reduced reactivity. These mechanistic effects put some limitations to the design of precursors suitable for rational synthesis of extended $\mathrm{sp}^{2}$ carbon systems. Additionally, steric hindrance in access to surface atoms within an extended molecular system may cause difficulties in activation of C-F bonds. Our rational design of precursor aimed to address all of the above considerations.

The cyclization of oligomers (2) was initiated through implementation of two $\mathrm{F}$ atoms placed in the same central anthracene unit of the precursor (Fig. 1C). Note that each of C$\mathrm{F}$ bond had a separate electron-rich anthracene system required for HF-elimination, and they occupied para positions within the same central anthracene block that provided the required configuration for effective cyclization. Moreover, because of the free rotation of single bonds between anthracene units, activation of all C-F bonds by surface Ti atoms was not limited by geometric constraints. Successful cyclodehydroflourination reactions were then followed by a series of programmed cyclodehydrogenations. In this case, we used structural-strain engineering within corresponding GNR intermediates, which, combined with lack of $\mathrm{TiO}_{2}$ catalytic activity for $\mathrm{H}_{2}$ elimination, provided high selectivity in formation of intramolecular C-C bonds.

As presented in Fig. 1C, intermediates (3) possessed highly-strained double helicene-like structures in single oligomer units, what facilitated closure of these two fjord-regions (30). These transformations finally led to formation of an oligomer composed of teranthene units (Fig. 1C, intermediate 4). As a result of high biradical character of teranthene fragments $(\mathrm{y}=42 \%)(36)$, we expected that further cyclodehydrogenation could violate the Woodward-Hoffmann rule given the low activation barrier (37-39). Thus, the resulting intermediate (4) should form target 7a-GNRs through a formally symmetry-forbidden thermal electrocyclization process.

On-surface synthesis procedures and low temperature STM characterization were performed in-situ under UHV conditions (for details see Supplementary Materials) to evaluate the reaction products. The rutile $\mathrm{TiO}_{2}$ monocrystals exposing (011) face were prepared by several sputtering and annealing cycles that led to a high-quality $(2 \times 1)$ reconstructed surface, as confirmed by low-energy electron diffraction (LEED) and STM imaging (see fig. S1, a and b). In order to induce the polymerization step, we deposited submonolayer coverages of DBDFTA molecules using a standard Knudsen cell on rutile (011) substrate kept at an elevated temperature of $\sim 470 \mathrm{~K}(22)$. Subsequent STM characterization revealed short oligoanthracene chains present on the reconstructed terraces as depicted in Fig. 2A and fig. S1d. Non-uniform STM contrast and apparent STM profile height of about $0.5 \mathrm{~nm}$ (Fig. 2B, red line) show flexibility between single anthracene units that gives rise to nonplanar geometry of the molecules.

By following our previously reported conditions for triggering C-F bond activation processes (30), we annealed the substrate at $\sim 670 \mathrm{~K}$. Corresponding large-scale STM images obtained after this step show straight and elongated features located mainly on chemically active sites such as terrace step edges or domain boundaries (Fig. $2 \mathrm{C}$ and fig. S2), which suggests successful on-surface synthesis of 7a-GNRs. To further verify this claim, we focused on single molecules on flat terraces. As shown in Fig. 2D, rigid $\mathrm{sp}^{2}$ carbon-based structure can be confirmed in this case by STM tip-induced manipulation (40). Application of a negative-bias voltage pulse resulted in rotation of the whole molecule without distorting its straight geometry. Moreover, this rotated molecule became flatter, as plotted in Fig. 2B (blue line), with 50\% reduction of the apparent height in comparison to oligoanthracene intermediate (2). The height contrast was now only dominated by both ends, proving the completed 
planarization of GNR.

The manipulation event also exposed native adsorption site of the GNR molecule, which was initially immobilized by surface hydroxyl groups. The presence of hydroxyl groups beneath newly formed GNRs (other examples in fig. S3) was consistent with previously reported manifestation of HFelimination reaction on the rutile $\mathrm{TiO}_{2}(011)$ (30). Our postulated GNR synthesis pathway shown in Fig. 1C was additionally confirmed in observation of the corresponding intermediates (4), which have not completed the last cyclodehydrogenation steps (see figs. S3 and S4).

Detailed analysis of end-states observed in most of the onsurface synthesized molecules could provide more insight into the exact morphology and electronic properties of finite 7a-GNRs on the rutile $\mathrm{TiO}_{2}$ substrate. A low-bias STM image (+0.9 V, Fig. 3A) showed a finite 7a-GNR comprised of six precursor units adsorbed on a domain boundary between two $(2 \times 1)$ reconstructed terraces. At these imaging conditions corresponding to unoccupied states around rutile $\mathrm{TiO}_{2}$ conduction band edge (CBE), both GNR ends exhibited characteristic three lobe features (see Fig. 3A, inset). This observation agreed with the expected symmetry of the lowest unoccupied molecular orbital (LUMO) for finite 7a-GNR terminated by two hydrogen-passivated zigzag ends, as captured by our spin-dependent density functional theory calculations (Fig. 3B) and corresponding unoccupied-states STM image simulations (Fig. 3C). Stability of zigzag ends was also consistent with previous reports on 7a-GNRs synthesized on metal substrates $(7,17,41)$.

Because the local electronic density of states related to LUMO resonance decayed in the central part of the GNR (see Fig. 3, B and C), the experimentally obtained STM image contrast was in this case determined by nonresonant tunneling through the molecule to the underlying substrate. Thus, it presented additional corrugation coming from the domain boundary structure. The good agreement between our STM results and gas-phase STM image simulations from Fig. 3C confirmed not only successful realization of the atomically precise GNR synthesis but also the weak coupling between obtained GNR and the rutile $\mathrm{TiO}_{2}$ substrate.

Moreover, calculated electronic structure presented in Fig. 3B revealed two spatially and energetically localized zigzag end states comprising of spin-polarized wave functions. Thus, the electronic ground state can be seen as two singleelectron occupied molecular orbitals reflecting open-shell character of the finite length 7a-GNR molecules. The antiferromagnetically coupled end-states offers a model system to study nonlocal spin entanglement, as proposed previously (32). However, with increasing length of GNRs the corresponding open-shell singlet to triplet excitation energy exponentially decayed to zero, and our examples of 7a-GNRs at 77 $\mathrm{K}$ became paramagnetic.
Characterization of such magnetic zig-zag end-states requires sufficient electronic decoupling from the substrate, which has been difficult to realize on reactive noble metals (42). Wang et al. (7) used insulating sodium chloride layers on $\mathrm{Au}(111)$ to decouple finite 7a-GNRs. Their systematic STS characterization showed that spectroscopic resonances of occupied and unoccupied end-localized states were split by energy-gap of $\Delta_{\mathrm{zz}}=1.9 \mathrm{eV}$. Because of intramolecular electronelectron correlation effects, self-consistent many-body perturbation theory calculations predict for free-standing finite 7a-GNRs $\Delta_{z z}$ of about $2.8 \mathrm{eV}(7)$. The $\sim 0.9 \mathrm{eV}$ difference in experimentally detected and calculated fundamental gaps was in that case explained by additional screening coming from electrons of the $\mathrm{NaCl} / \mathrm{Au}(111)$ substrate.

Figure 4 shows our STS differential conductance $d I / d V$ data obtained on finite 7a-GNRs adsorbed on the rutile $\mathrm{TiO}_{2}$ (see also fig. S5). Two zigzag end resonances (red curve) were localized within the energy gap of bulk armchair GNR states (blue curve), in excellent agreement with previous reports (7). Interestingly, the measured quasi-particle gap $\Delta_{\mathrm{zz}}=2.45 \pm 0.10$ $\mathrm{eV}$ was near the values expected for the free-standing system, indicating minimal interaction with the substrate electrons. A strong intensity asymmetry between registered resonances came from the difference in their relative energy positions with respect to the rutile $\mathrm{TiO}_{2}$ electronic structure. An unoccupied edge state at $+0.9 \mathrm{~V}$ was located near the CBE of the substrate. In contrary, occupied edge state at $-1.45 \mathrm{~V}$ fell into a wide $\mathrm{TiO}_{2}$ bandgap that caused sharp, Lorentzian-like shape of the corresponding $d I / d V$ resonance.

Our results show that the magnetic ground state of the synthetized model open-shell GNRs was electronically decoupled from the substrate, which opens future possibilities for probing their intrinsic transport properties $(43,44)$. Moreover, recent developments of scanning probe microscopes (SPMs) compatible with the microwave transmission have demonstrated detection of single electron spin resonances $(45,46)$. Application of these techniques to decoupled spin centers in graphitic molecular systems would enable the complete processing of quantum information including qubit initialization, logic gate operation, and readout $(10,47)$. Finally, atom-manipulation protocols and multiprobe SPMs could further advance these efforts toward more complex quantum circuitry (40).

\section{REFERENCES AND NOTES}

1. L. Talirz, P. Ruffieux, R. Fasel, On-Surface Synthesis of Atomically Precise Graphene Nanoribbons. Adv. Mater. 28, 6222-6231 (2016). doi:10.1002/adma.201505738 Medline

2. K. Müllen, X. Feng, From Polyphenylenes to Nanographenes and Graphene Nanoribbons. Advances in Polymer Science (Springer International Publishing, 2017).

3. T. Cao, F. Zhao, S. G. Louie, Topological Phases in Graphene Nanoribbons: Junction States, Spin Centers, and Quantum Spin Chains. Phys. Rev. Lett. 119, 076401 (2017). doi:10.1103/PhysRevLett.119.076401 Medline

4. O. Gröning, S. Wang, X. Yao, C. A. Pignedoli, G. Borin Barin, C. Daniels, A. Cupo, V. 
Meunier, X. Feng, A. Narita, K. Müllen, P. Ruffieux, R. Fasel, Engineering of robust topological quantum phases in graphene nanoribbons. Nature 560, 209-213 (2018). doi:10.1038/s41586-018-0375-9 Medline

5. D. J. Rizzo, G. Veber, T. Cao, C. Bronner, T. Chen, F. Zhao, H. Rodriguez, S. G. Louie, M. F. Crommie, F. R. Fischer, Topological band engineering of graphene nanoribbons. Nature 560, 204-208 (2018). doi:10.1038/s41586-018-0376-8 Medline

6. P. Ruffieux, S. Wang, B. Yang, C. Sánchez-Sánchez, J. Liu, T. Dienel, L. Talirz, P. Shinde, C. A. Pignedoli, D. Passerone, T. Dumslaff, X. Feng, K. Müllen, R. Fasel, Onsurface synthesis of graphene nanoribbons with zigzag edge topology. Nature 531, 489-492 (2016). doi:10.1038/nature17151 Medline

7. S. Wang, L. Talirz, C. A. Pignedoli, X. Feng, K. Müllen, R. Fasel, P. Ruffieux, Giant edge state splitting at atomically precise graphene zigzag edges. Nat. Commun. 7, 11507 (2016). doi:10.1038/ncomms 11507 Medline

8. S. Mishra, D. Beyer, R. Berger, J. Liu, O. Gröning, J. I. Urgel, K. Müllen, P. Ruffieux, X. Feng, R. Fasel, Topological Defect-Induced Magnetism in a Nanographene. J. Am. Chem. Soc. 142, 1147-1152 (2020). doi:10.1021/jacs.9b09212 Medline

9. J. Li, S. Sanz, M. Corso, D. J. Choi, D. Peña, T. Frederiksen, J. I. Pascual, Single spin localization and manipulation in graphene open-shell nanostructures. Nat. Commun. 10, 200 (2019). doi:10.1038/s41467-018-08060-6 Medline

10. F. Lombardi, A. Lodi, J. Ma, J. Liu, M. Slota, A. Narita, W. K. Myers, K. Müllen, X. Feng, L. Bogani, Quantum units from the topological engineering of molecular graphenoids. Science 366, 1107-1110 (2019). doi:10.1126/science.aay7203 Medline

11. S. Mishra, D. Beyer, K. Eimre, S. Kezilebieke, R. Berger, O. Gröning, C. A. Pignedoli, K. Müllen, P. Liljeroth, P. Ruffieux, X. Feng, R. Fasel, Topological frustration induces unconventional magnetism in a nanographene. Nat. Nanotechnol. 15, 2228 (2020). Medline

12. S. von Kugelgen, D. E. Freedman, A chemical path to quantum information. Science 366, 1070-1071 (2019). doi:10.1126/science.aaz4044 Medline

13. S. Clair, D. G. de Oteyza, Controlling a Chemical Coupling Reaction on a Surface: Tools and Strategies for On-Surface Synthesis. Chem. Rev. 119, 4717-4776 (2019). doi:10.1021/acs.chemrev.8b00601 Medline

14. X. Zhou, G. Yu, Modified Engineering of Graphene Nanoribbons Prepared via OnSurface Synthesis. Adv. Mater. 32, e1905957, 1905957 (2019). Medline

15. M. Treier, C. A. Pignedoli, T. Laino, R. Rieger, K. Müllen, D. Passerone, R. Fasel, Surface-assisted cyclodehydrogenation provides a synthetic route towards easily processable and chemically tailored nanographenes. Nat. Chem. 3, 61-67 (2011). doi:10.1038/nchem.891 Medline

16. J. Cai, P. Ruffieux, R. Jaafar, M. Bieri, T. Braun, S. Blankenburg, M. Muoth, A. P. Seitsonen, M. Saleh, X. Feng, K. Müllen, R. Fasel, Atomically precise bottom-up fabrication of graphene nanoribbons. Nature 466, 470-473 (2010). doi:10.1038/nature09211 Medline

17. P. H. Jacobse, M. J. J. Mangnus, S. J. M. Zevenhuizen, I. Swart, Mapping the Conductance of Electronically Decoupled Graphene Nanoribbons. ACS Nano 12 , 7048-7056 (2018). doi:10.1021/acsnano.8b02770 Medline

18. O. Deniz, C. Sánchez-Sánchez, T. Dumslaff, X. Feng, A. Narita, K. Müllen, N. Kharche, V. Meunier, R. Fasel, P. Ruffieux, Revealing the Electronic Structure of Silicon Intercalated Armchair Graphene Nanoribbons by Scanning Tunneling Spectroscopy. Nano Lett. 17, 2197-2203 (2017). doi:10.1021/acs.nanolett.6b04727 Medline

19. J. P. Llinas, A. Fairbrother, G. Borin Barin, W. Shi, K. Lee, S. Wu, B. Yong Choi, R. Braganza, J. Lear, N. Kau, W. Choi, C. Chen, Z. Pedramrazi, T. Dumslaff, A. Narita, X. Feng, K. Müllen, F. Fischer, A. Zettl, P. Ruffieux, E. Yablonovitch, M. Crommie, R. Fasel, J. Bokor, Short-channel field-effect transistors with 9-atom and 13-atom wide graphene nanoribbons. Nat. Commun. 8, 633 (2017). doi:10.1038/s41467017-00734-x Medline

20. G. Borin Barin, A. Fairbrother, L. Rotach, M. Bayle, M. Paillet, L. Liang, V. Meunier, R. Hauert, T. Dumslaff, A. Narita, K. Müllen, H. Sahabudeen, R. Berger, X. Feng, R. Fasel, P. Ruffieux, Surface-Synthesized Graphene Nanoribbons for Room Temperature Switching Devices: Substrate Transfer and ex Situ Characterization. ACS Appl. Nano Mater. 2, 2184-2192 (2019). doi:10.1021/acsanm.9b00151

21. A. Radocea, T. Sun, T. H. Vo, A. Sinitskii, N. R. Aluru, J. W. Lyding, SolutionSynthesized Chevron Graphene Nanoribbons Exfoliated onto H:Si(100). Nano Lett. 17, 170-178 (2017). doi:10.1021/acs,nanolett.6b03709 Medline
22. M. Kolmer, A. A. Ahmad Zebari, J. S. Prauzner-Bechcicki, W. Piskorz, F. Zasada, S. Godlewski, B. Such, Z. Sojka, M. Szymonski, Polymerization of polyanthrylene on a titanium dioxide (011)-(2×1) surface. Angew. Chem. Int. Ed. 52, 10300-10303 (2013). doi:10.1002/anie.201303657 Medline

23. M. Kolmer, R. Zuzak, A. A. Ahmad Zebari, S. Godlewski, J. S. Prauzner-Bechcicki, W. Piskorz, F. Zasada, Z. Sojka, D. Bléger, S. Hecht, M. Szymonski, On-surface polymerization on a semiconducting oxide: Aryl halide coupling controlled by surface hydroxyl groups on rutile Ti02(011). Chem. Commun. 51, 11276-11279 (2015). doi:10.1039/C5CC02989A Medline

24. G. Vasseur, M. Abadia, L. A. Miccio, J. Brede, A. Garcia-Lekue, D. G. de Oteyza, C. Rogero, J. Lobo-Checa, J. E. Ortega, $\Pi$ Band Dispersion along Conjugated Organic Nanowires Synthesized on a Metal Oxide Semiconductor. J. Am. Chem. Soc. 138, 5685-5692 (2016). doi:10.1021/jacs.6b02151 Medline

25. K. Y. Amsharov, M. A. Kabdulov, M. Jansen, Facile bucky-bowl synthesis by regiospecific cove-region closure by HF elimination. Angew. Chem. Int. Ed. 51 , 4594-4597 (2012). doi:10.1002/anie.201200516 Medline

26. K. Y. Amsharov, P. Merz, Intramolecular aryl-aryl coupling of fluoroarenes through Al203-mediated HF elimination. J. Org. Chem. 77, 5445-5448 (2012) doi:10.1021/j0300783y Medline

27. K. Amsharov, Cyclodehydrofluorination of fluoroarenes on metal oxides: Toward bottom-up synthesis of carbon nanostructures on insulating surfaces. Phys. Status Solidi, B Basic Res. 253, 2473-2477 (2016). doi:10.1002/pssb.201600211

28. A. K. Steiner, K. Y. Amsharov, The Rolling-Up of Oligophenylenes to Nanographenes by a HF-Zipping Approach. Angew. Chem. Int. Ed. 56, 1473214736 (2017). doi:10.1002/anie.201707272 Medline

29. D. Sharapa, A. K. Steiner, K. Amsharov, The Mechanism of Cyclodehydrofluorination on gamma-Alumina. Phys. Status Solidi, B Basic Res. 255, 1800189 (2018). doi:10.1002/pssb.201800189

30. M. Kolmer, R. Zuzak, A. K. Steiner, L. Zajac, M. Engelund, S. Godlewski, M. Szymonski, K. Amsharov, Fluorine-programmed nanozipping to tailored nanographenes on rutile $\mathrm{TiO}_{2}$ surfaces. Science 363, 57-60 (2019). doi:10.1126/science. aav4954 Medline

31. L. Talirz, H. Söde, J. Cai, P. Ruffieux, S. Blankenburg, R. Jafaar, R. Berger, X. Feng, K. Müllen, D. Passerone, R. Fasel, C. A. Pignedoli, Termini of bottom-up fabricated graphene nanoribbons. J. Am. Chem. Soc. 135, 2060-2063 (2013). doi:10.1021/ja311099k Medline

32. M. Golor, C. Koop, T. C. Lang, S. Wessel, M. J. Schmidt, Magnetic correlations in short and narrow graphene armchair nanoribbons. Phys. Rev. Lett. 111, 085504 (2013). doi:10.1103/PhysRevLett.111.085504 Medline

33. L. Benz, J. Haubrich, R. G. Quiller, S. C. Jensen, C. M. Friend, McMurry chemistry on $\mathrm{TiO}(2)(110)$ : Reductive $\mathrm{C}=\mathrm{C}$ coupling of benzaldehyde driven by titanium interstitials. J. Am. Chem. Soc. 131, 15026-15031 (2009). doi:10.1021/ja905522c Medline

34. V. Akhmetov, M. Feofanov, S. Troyanov, K. Amsharov, Tailoring Diindenochrysene through Intramolecular Multi-Assemblies by C-F Bond Activation on Aluminum Oxide. Chemistry 25, 7607-7612 (2019). doi:10.1002/chem.201901450 Medline

35. V. Akhmetov, M. Feofanov, O. Papaianina, S. Troyanov, K. Amsharov, Towards Nonalternant Nanographenes through Self-Promoted Intramolecular Indenoannulation Cascade by C-F Bond Activation. Chemistry 25, 11609-11613 (2019). doi:10.1002/chem.201902586 Medline

36. A. Konishi, Y. Hirao, M. Nakano, A. Shimizu, E. Botek, B. Champagne, D. Shiomi, K Sato, T. Takui, K. Matsumoto, H. Kurata, T. Kubo, Synthesis and characterization of teranthene: A singlet biradical polycyclic aromatic hydrocarbon having Kekulé structures. J. Am. Chem. Soc. 132, 11021-11023 (2010). doi:10.1021/ja1049737 Medline

37. T. Šolomek, P. Ravat, Z. Mou, M. Kertesz, M. Juríček, Cethrene: The Chameleon of Woodward-Hoffmann Rules. J. Org. Chem. 83, 4769-4774 (2018). doi:10.1021/acs.joc.8b00656 Medline

38. T. Šolomek, P. Ravat, M. Juríček, 'Forbidden' Electrocyclizations of Diradicaloids. Trends Chem. 1, 705-706 (2019). doi:10.1016/i.trechm.2019.08.005

39. P. Ravat, T. Šolomek, D. Häussinger, O. Blacque, M. Juríček, Dimethylcethrene: A Chiroptical Diradicaloid Photoswitch. J. Am. Chem. Soc. 140, 10839-10847 (2018). doi:10.1021/jacs.8b05465 Medline

40. W. Ko, C. X. Ma, G. D. Nguyen, M. Kolmer, A. P. Li, Atomic-Scale Manipulation and In Situ Characterization with Scanning Tunneling Microscopy. Adv. Funct. Mater. 
29, 1903770 (2019). doi:10.1002/adfm.201903770

41. M. Koch, F. Ample, C. Joachim, L. Grill, Voltage-dependent conductance of a single graphene nanoribbon. Nat. Nanotechnol. 7, 713-717 (2012). doi:10.1038/nnano.2012.169 Medline

42. Y. Li, W. Zhang, M. Morgenstern, R. Mazzarello, Electronic and magnetic properties of zigzag graphene nanoribbons on the (111) surface of $\mathrm{Cu}, \mathrm{Ag}$, and Au. Phys. Rev. Lett. 110, 216804 (2013). doi:10.1103/PhysRevLett.110.216804 Medline

43. M. Kolmer, P. Brandimarte, J. Lis, R. Zuzak, S. Godlewski, H. Kawai, A. GarciaLekue, N. Lorente, T. Frederiksen, C. Joachim, D. Sanchez-Portal, M. Szymonski, Electronic transport in planar atomic-scale structures measured by two-probe scanning tunneling spectroscopy. Nat. Commun. 10, 1573 (2019). doi:10.1038/s41467-019-09315-6 Medline

44. A. L. Miettinen, M. S. Nevius, W. Ko, M. Kolmer, A.-P. Li, M. N. Nair, B. Kierren, L. Moreau, E. H. Conrad, A. Tejeda, Edge states and ballistic transport in zigzag graphene ribbons: The role of SiC polytypes. Phys. Rev. B 100, 045425 (2019). doi:10.1103/PhysRevB.100.045425

45. F. D. Natterer, K. Yang, W. Paul, P. Willke, T. Choi, T. Greber, A. J. Heinrich, C. P. Lutz, Reading and writing single-atom magnets. Nature 543, 226-228 (2017). doi:10.1038/nature21371 Medline

46. P. Willke, K. Yang, Y. Bae, A. J. Heinrich, C. P. Lutz, Magnetic resonance imaging of single atoms on a surface. Nat. Phys. 15, 1005-1010 (2019). doi:10.1038/s41567019-0573-x

47. K. Yang, W. Paul, S.-H. Phark, P. Willke, Y. Bae, T. Choi, T. Esat, A. Ardavan, A. J. Heinrich, C. P. Lutz, Coherent spin manipulation of individual atoms on a surface. Science 366, 509-512 (2019). doi:10.1126/science.aay6779 Medline

48. J. M. Soler, E. Artacho, J. D. Gale, A. García, J. Junquera, P. Ordejón, D. SánchezPortal, The SIESTA method for ab initio order-N materials simulation. J. Phys. Condens. Matter 14, 2745-2779 (2002). doi:10.1088/0953-8984/14/11/302

49. J. P. Perdew, K. Burke, M. Ernzerhof, Generalized gradient approximation made simple. Phys. Rev. Lett. 77, 3865-3868 (1996). doi:10.1103/PhysRevLett.77.3865 Medline

50. 0. Paz, J. M. Soler, Efficient and reliable method for the simulation of scanning tunneling images and spectra with local basis sets. Phys. Status Solidi, B Basic Res. 243, 1080-1094 (2006). doi:10.1002/pssb.200541453

51. J. Tersoff, D. R. Hamann, Theory and Application for the Scanning Tunneling Microscope. Phys. Rev. Lett. 50, 1998-2001 (1983). doi:10.1103/PhysRevLett.50.1998

52. A. P. Krapcho, Z. Getahun, Convenient Synthetic Routes to 1,4Difluoroanthracene-9,10-Dione. Synth. Commun. 15, 907-910 (1985). doi:10.1080/00397918508063889

\section{ACKNOWLEDGMENTS}

Funding: This work was supported in part by the Center for Nanophase Materials Sciences (CNMS), which is a DOE Office of Science User Facility (M.K., W.K., A.P.L.). Research was partially funded by grant ONR N00014-16-1-3213 and ONR N00014-20-1-2302 at the University of Tennessee (W.K., A.-P.L.). Preliminary research was supported by the Polish Ministry of Science and Higher Education, contract no. 0341/IP3/2016/74 (I.I.). A.K.S and K.A thank the Deutsche Forschungsgemeinschaft for financial support (Projektnummer 182849149-SFB 953 A6 and AM407). Author contributions: M.K., A.P.L., and K.A. conceived the project. K.A. and A.K.S. carried out the precursor synthesis and analysis. M.K. conducted the on-surface synthesis and characterization experiments with support from W.K, and A.P.L. M.K., I.I. and M.S. performed the preliminary experiments. M.E. conducted the computations. M.K., A.-P.L. and K.A. wrote the manuscript with feedback from all other authors; Competing interests: Authors declare no competing interests; Data and materials availability: All data are available in the main text or the supplementary materials.

\section{SUPPLEMENTARY MATERIALS}

science.sciencemag.org/cgi/content/full/science.abb8880/DC1

Materials and Methods

Figs. S1 to S13

References (48-52)
25 March 2020; accepted 3 June 2020

Published online 25 June 2020

10.1126/science.abb8880 
A Au(111) Cai et al. 2010

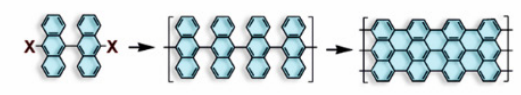

B $\quad \mathrm{TiO}_{2}$ Kolmer et al. 2019

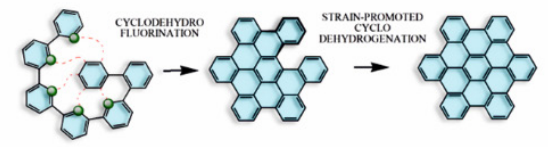

$(0=$ C.F)

C

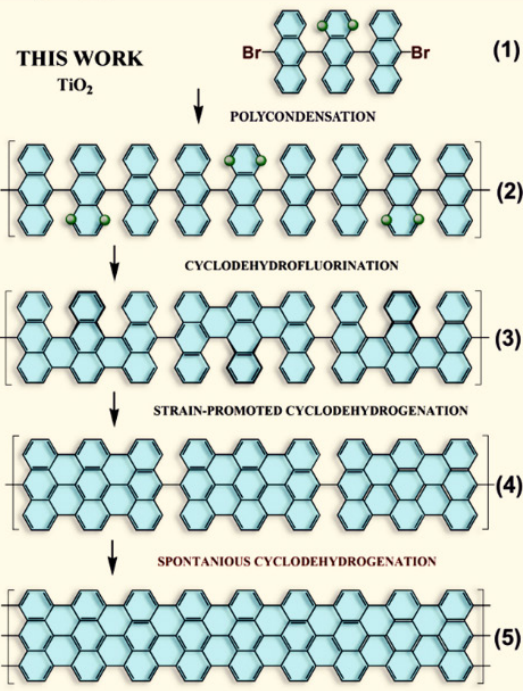

Fig. 1. On-surface synthesis strategies for nanographenes formation. (A) Scheme of the most common two-step strategy of graphene nanoribbons formation introduced by Cai et al. on noble metal substrates (16). X represents halogen atom (typically $\mathrm{Br}$ or I). (B and $\mathrm{C}$ ) Multistep metal oxide surface-assisted strategies for formation of compact nanographenes [(B), from (30)] and extended graphene nanoribbons $[(\mathrm{C})$, this work]. $\mathrm{C}-\mathrm{F}$ bonds are shown as green circles for clarity 

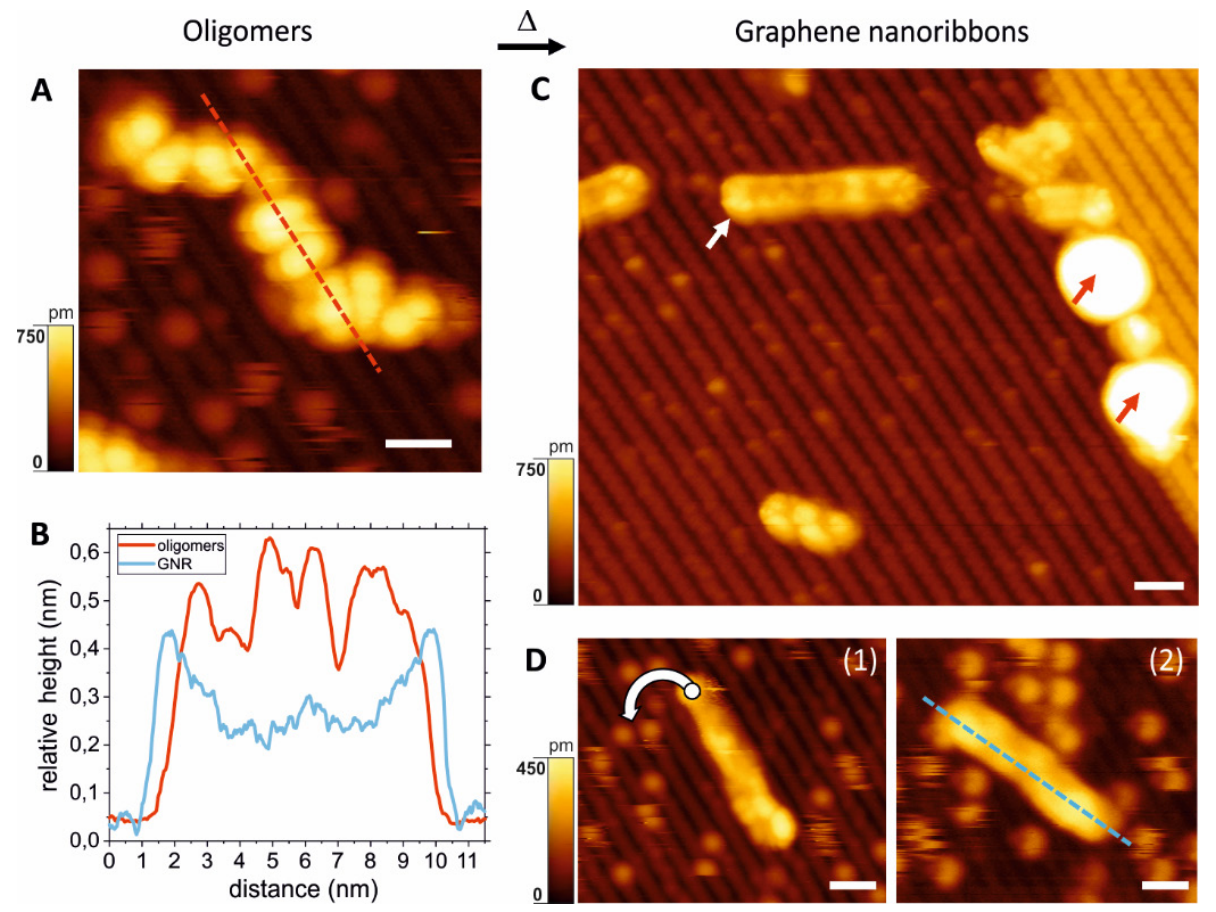

Fig. 2. Transformation of oligoanthracenes into graphene nanoribbons. (A) STM image ( $+3 \mathrm{~V} ; 2 \mathrm{pA}$ ) of oligoanthracenes obtained after DBDFTA deposition on the rutile (011)- $(2 \times 1)$ kept at $470 \mathrm{~K}$. (B) Cross sections along lines in panels a (red) and d (blue) obtained in comparable STM imaging conditions. (C) STM image (+2.5 V; $10 \mathrm{pA}$ ) of 7 armchair graphene nanoribbon (7a-GNR) obtained after DBDFTA deposition on the rutile (011)- $(2 \times 1)$ kept at $470 \mathrm{~K}$ followed by annealing at $670 \mathrm{~K}$. White arrow marks about $9 \mathrm{~nm}$ long GNR adsorbed on a domain boundary. Observed defects are related to polycondensation reaction by-products (red arrows). (D) Two sequential STM images (1; $+2 \mathrm{~V}, 5 \mathrm{pA})$ and $(2 ;+3 \mathrm{~V} ; 2 \mathrm{pA})$ of the same $7 \mathrm{a}-\mathrm{GNR}$ on a flat terrace. GNR is rotated by STM bias voltage pulse $-3 \vee$ applied in location marked by white circle in (1). Note that initial adsorption site observed in (2) is covered by surface hydroxyl groups. Scale bars in all STM images are $2 \mathrm{~nm}$. 

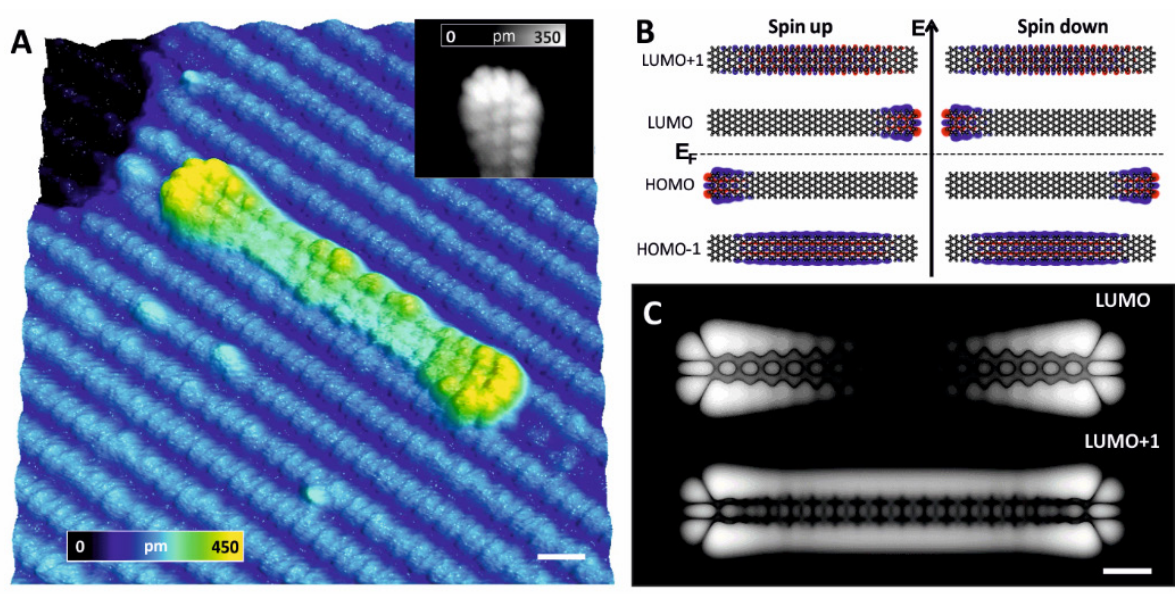

Fig. 3. Spin-polarized end states in finite 7a-GNRs. (A) 3D visualization of high resolution STM image (+0.9 V; $2 \mathrm{pA})$ presenting finite 7a-GNR synthesized from six DBDFTA precursors with clearly symmetric edge electronic states (see inset). GNR is adsorbed on a domain boundary between two reconstructed rutile (011)-(2×1) terraces (blue color). Black region in the corner is the lower-laying terrace. (B) Real space visualization of spin-polarized electronic wave functions for finite 7a-GNR synthesized from six DBDFTA precursors and terminated by perfect ZZ edges. Two occupied and unoccupied electronic states in single-electron molecular orbitals correspond to $\mathrm{HOMO}+1, \mathrm{HOMO}, \mathrm{LUMO}$ and LUMO+1, respectively. Note that symmetric HOMO and LUMO states represent in this case spin-polarized zigzag edge states of 7a-GNR. (C) STM image simulation obtained for bias voltage corresponding to the first and second excited unoccupied state (LUMO and LUMO+1). Scale bars in A and $C$ are $1 \mathrm{~nm}$. 

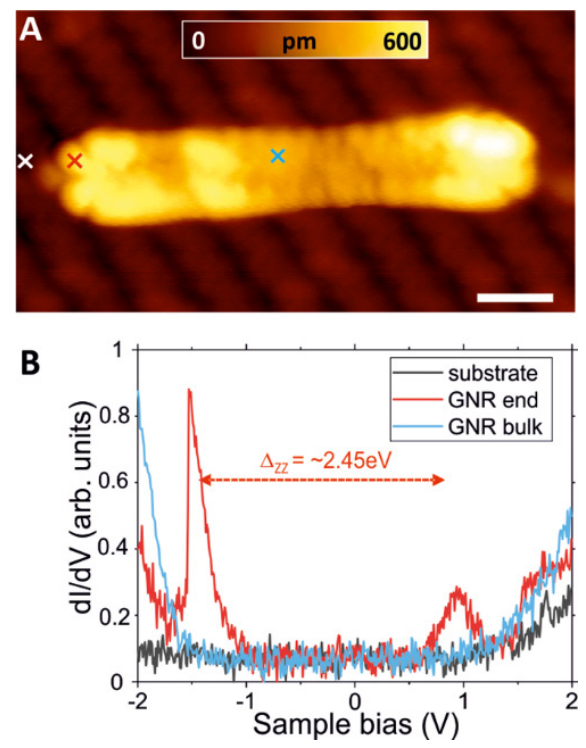

Fig. 4. Electronic structure characterization. (A) High-resolution STM image (+1.5 V, $10 \mathrm{pA})$ of 7a-GNR synthesized on the rutile (011). Molecule is adsorbed on a domain boundary between two reconstructed rutile (011)-(2×1) terraces (see also Fig. $2 b)$. Scale bar is $1 \mathrm{~nm}$. (B) Scanning tunneling spectroscopy $d l / d V$ data obtained in locations marked by three crosses in a. Two resonances marked by red arrow at about $-1.45 \mathrm{eV}$ and $+1.0 \mathrm{eV}$ correspond to occupied and unoccupied zigzag end state, respectively 